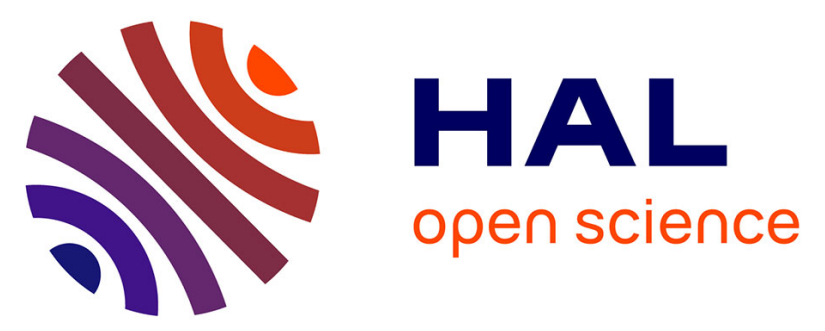

\title{
Electron Channeling Contrast Imaging characterization and crystal plasticity modelling of dislocation activity in Ti21S BCC material
}

\author{
Meriem Ben Haj Slama, Vincent Taupin, Nabila Maloufi, Kaustubh \\ Venkatraman, Anthony Rollett, Ricardo A Lebensohn, Stephane Berbenni, \\ Benoît Beausir, Antoine Guitton
}

\section{To cite this version:}

Meriem Ben Haj Slama, Vincent Taupin, Nabila Maloufi, Kaustubh Venkatraman, Anthony Rollett, et al.. Electron Channeling Contrast Imaging characterization and crystal plasticity modelling of dislocation activity in Ti21S BCC material. Materialia, 2021, 15, pp.100996. 10.1016/j.mtla.2020.100996 . hal-03094460

\section{HAL Id: hal-03094460 \\ https://hal.univ-lorraine.fr/hal-03094460}

Submitted on 9 Jan 2021

HAL is a multi-disciplinary open access archive for the deposit and dissemination of scientific research documents, whether they are published or not. The documents may come from teaching and research institutions in France or abroad, or from public or private research centers.
L'archive ouverte pluridisciplinaire HAL, est destinée au dépôt et à la diffusion de documents scientifiques de niveau recherche, publiés ou non, émanant des établissements d'enseignement et de recherche français ou étrangers, des laboratoires publics ou privés.

\section{(ㅇ)(1) $\$$}

Distributed under a Creative Commons Attribution - NonCommercial - NoDerivatives 44.0 


\title{
Electron channeling contrast imaging characterization and crystal plasticity modelling of dislocation activity in Ti21S BCC material
}

\author{
Meriem Ben Haj Slama a,b, Vincent Taupin ${ }^{\mathrm{a}, \mathrm{b}}$, Nabila Maloufi ${ }^{\mathrm{a}, \mathrm{b}}$, Kaustubh Venkatraman ${ }^{\mathrm{a}, \mathrm{b}}$, \\ Anthony D. Rollett ${ }^{\mathrm{b}, \mathrm{c}}$, Ricardo A. Lebensohn ${ }^{\mathrm{d}}$, Stéphane Berbenni ${ }^{\mathrm{a}}{ }^{\mathrm{a}}$, Benoît Beausir ${ }^{\mathrm{a}, \mathrm{b}}$, \\ Antoine Guitton ${ }^{\mathrm{a}, \mathrm{b}, *}$ \\ a Université de Lorraine - CNRS - Arts et Métiers ParisTech - LEM3, France \\ ${ }^{\mathrm{b}}$ Labex Damas - Université de Lorraine, France \\ ${ }^{\mathrm{c}}$ Department of Materials Science and Engineering, Carnegie Mellon University, 5000 Forbes Ave, Pittsburgh, PA 15213, USA \\ d Theoretical Division, Los Alamos National Laboratory, Los Alamos, NM 87845, USA
}

\section{A R T I C L E I N F O}

\section{Keywords:}

In situ

Tensile test

Crystal plasticity

ECCI

Slip-system

BCC titanium

\begin{abstract}
A B S T R A C T
In this paper, an original approach is proposed to compare modeling and relevant statistical experiments using $\beta$-Ti21S Body Centered Cubic (BCC) metal as a challenging benchmark. Our procedure allows the evolution of microstructural defects to be tracked in situ with excellent spatial resolution, while observing a bulk sample region sufficiently large to be statistically representative of the material. We identify multiple mechanisms such as slip transfer, slip traces, pencil glide, etc. We demonstrate that for small plastic strains $(<0.25 \%)$ under uniaxial tensile loading, the Schmid law is satisfied statistically. Under these circumstances, changes in Critical Resolved Shear Stress (CRSS) are minimal and accommodation of incompatible deformation between grains has not yet become important. The majority of the observed slip plane traces at the mesoscale corresponds to the $\{123\}$ family. Fully automated while precise, the reported approach compares this data with four crystal plasticity models, and provides a methodology for similar analyses in other materials.
\end{abstract}

\section{Introduction}

In metals, elementary mechanisms such as dislocation glide, operate at the atomic and electronic scale; typically, the Ångström and the picosecond. They can strongly influence the macroscopic strength at the laboratory scale, e.g. the centimeter and the second. For example, $a b$ initio simulations of dislocation cores in Body Centered Cubic (BCC) metals highlighted the occurrence of non-Schmid effects, which could explain asymmetry between tension and compression at the macroscopic scale $[1-3]$.

To understand and ultimately control the mechanical behavior of metallic materials with regards to their processed microstructures, one has to face this impressively complex multiscale problem that can span more than 10 orders of magnitude in time and space. This is why advanced theoretical and experimental tools are constantly being developed, aiming at bridging relevant length scales and at understanding the mechanisms involved.

In support of these aims, experimental methods include several imaging (High-Resolution Transmission HR-TEM, Scanning Tunneling STM, Atomic Force Microscopy AFM) and diffraction techniques (High-Energy

\footnotetext{
* Corresponding author.

E-mail address: antoine.guitton@univ-lorraine.fr (A. Guitton).
}

X-Ray Diffraction HR-XRD, Electron BackScattered Diffraction EBSD...) that provide valuable atomic scale information, such as activation energies and critical shear stresses. However, one may argue that most multiscale strategies usually address only very specific cases, and that they are not necessarily statistically representative of the myriad of mechanisms that can occur in real polycrystals. For instance, one can cite HR-TEM [4] studies of special grain boundaries (structure, energy, migration under stress and temperature...) and their interactions with dislocations (emission, absorption, transmission...). Indeed, such available studies are far from covering the full range of possible grain boundary configurations in a real polycrystal. In addition, preparing and testing of thin foils for electron transmission-based techniques [5-7] (TEM, Transmission Kikuchi Diffraction TKD) can unfortunately affect the defect microstructure and the mechanisms investigated because of inherent size, strain rate, and external surface effects. Besides, these techniques do not provide enough data for statistical analysis of physical mechanisms.

Experimental findings at an intermediate, mesoscopic scale, can be used to improve our knowledge of polycrystal plasticity and to improve models at the grain scale. They provide validation data for advanced numerical simulations that predict the mechanical behavior of polycrystals (strain hardening, ductility etc.), with respect to the evolution of disloca- 
tion densities inside the grains and their interactions with grain boundaries. Modeling tools include Discrete Dislocation Dynamics (DDD), Crystal Plasticity (CP) models based on Finite Elements or Fast Fourier Transform algorithms (CPFEM, CPFFT), or mean-field approaches (e.g., self-consistent models such as the ViscoPlastic Self-Consistent (VPSC), and Elasto-Visco-Plastic Self-Consistent (EVPSC) schemes) [8]. Recent studies have been carried out that aim to couple models to experiments such as CPFEM (or CPFFT) with EBSD [9-11], or CPFEM with High-Resolution Digital Image Correlation HRDIC [12]. Such coupled approaches can provide information on textures, Geometrically Necessary Dislocation (GND) density patterning, plastic strain localization and transfer across grain boundaries, etc. Among the results, such coupled modeling-experimental methods show that it is possible to link the microstructural evolution to stress-strain behavior.

However, the majority of models revealed that each configuration usually has its own behavior, especially concerning dislocation interactions, such that a huge number of simulations are required to characterize all possible mechanisms. Perhaps more importantly, the analysis of individual "model" configurations, by means of molecular dynamics for example $[13,14]$ although very instructive, usually do not or cannot consider the non-local influence of the surrounding microstructure on the local mechanisms investigated. For instance, the activation of a particular dislocation system in a grain or the interaction of a dislocation with a grain boundary are obviously strongly dependent on the elastic fields induced by nearby grains, grain boundaries, triple lines and other possible defects such as solutes or precipitates.

A few studies have tried to make an overview of constitutive laws and theories for crystal plasticity modelling $[15,16]$, but to our knowledge, none of them provided quantitative and statistical comparisons between these several modelling methods that could shed new light on the inherent mechanisms. In this paper, a combined experimental/theoretical approach is proposed to fill this gap.

Concerning our experimental approach, the strategy reported in this paper is based on Scanning Electron Microscope (SEM) measurements. SEM is flexible essentially because of the large sample surface area that can be imaged, as compared to TEM. Moreover, in SEM, the nondestructive Electron Channeling Contrast Imaging ECCI imaging mode provides TEM-like diffraction contrast imaging of near-surface defects with a good accuracy [17-21]. In terms of length scales, centimetric bulk materials can be characterized, enabling statistical studies. A more powerful technique, Accurate Electron Channeling Contrast Imaging (AECCI) developed some years ago [21], has an angular accuracy better than $0.1^{\circ}$ with a spatial resolution finer than $500 \mathrm{~nm}$ [22]. By coupling A-ECCI to an in situ tensile test inside the SEM [23], several areas on a bulk sample can be accessed with a single tool while under mechanical load, thus covering the scale from sample to dislocations. The availability of multiple regions of interest is helpful in enumerating relevant statistical parameters from the mesoscopic and microscopic descriptions of microstructures, which in turn provides large enough datasets for data mining.

For numerical calculations, we consider three mean-field models and a full-field CPFFT model. The first mean field model uses the Sachs assumption, i.e., that the local stress state is equal to the macroscopic one [24]. This assumption is commonly made in experimental characterization of dislocation activity, where uniform stress is applicable in contrast to the uniform strain assumed in the Taylor model [25], which is more successful for texture prediction at large strain. Hence, our hypothesis is that the uniform stress model is more reliable than others, at low plastic strains, for a material with many possible slip systems. In addition, we use two popular models, i.e., the VPSC model [26,27], a well-known model for prediction of texture development in polycrystals, and a more recent affine-type EVPSC model based on the translated field method by Mareau and Berbenni [28]. The affine-type EVPSC model was recently applied to study the effect of anisotropic elasticity stiffness components $\left(\mathrm{C}_{11}, \mathrm{C}_{12}, \mathrm{C}_{44}\right)$ on the macroscopic behavior of polycrystalline $\beta$-Ti alloys, especially the transition between elastic and plastic states, and the development of incompatibility stresses (i.e., inter-granular internal stresses) within the polycrystal [29]. The fourth full-field model (CPFFT) is an elasto-viscoplastic formulation with anisotropic elasticity/plasticity [30,31]. The different model predictions are compared with experimental data in terms of slip system activities at the onset of plasticity. For the majority of metals, slip activation at yield follows the well-known Schmid law [32-34] and the expected results are that: (i) the slip system with the highest Resolved Shear Stress (RSS) is activated (before others) and (ii) the RSS of the activated slip system is necessarily equal to or higher than its associated Critical Resolved Shear stress (CRSS).

Marked by an atypical and still not well understood plastic behavior, BCC metals are reported to clearly violate this law [34]. Theoretical calculations showed that the CRSS of the three BCC slip families depend on the crystal orientation with respect to the loading direction [35]. More recent work proposed a modified parameter-free Schmid law to quantify the deviations from the Schmid law based on the deviations of the screw dislocation trajectory away from a straight path between equilibrium configurations [36]. This deviation from the Schmid law is metal-dependent [37]. Furthermore, the number of activated slip systems in BCC metals can be as high as 48, and "pencil glide" (see §.3.1) is frequently reported in experiments. For those reasons, we choose a BCC metal as a challenging benchmark for our proposed coupled experimental / theoretical strategy, i.e., $\beta$-Ti21S. Note further that elastic anisotropy is important in this material [38], making the analysis more complex, even at the onset of plasticity.

In what follows, we present the development of an experimentaltheoretical approach that allows comparing several modeling methods while contrasting their results with experiment.

The experimental work that we describe below is driven by the following objectives: (i) perform observations on a bulk specimen, (ii) provide an in situ observation of dislocations and slip lines during deformation, (iii) characterize a large polycrystalline area where hundreds of plastic events (dislocations and slip lines) can be captured, (iv) conduct an automated statistical analysis of the data collected.

Modeling with several crystal plasticity theories enables (i), documentation and quantification of the long-range influence of internal stresses due to neighboring grains and defects on the local mechanisms observed and (ii), the use of statistical analysis to improve mechanical models.

Comparison with simulations is assisted by an automated analysis of the observed microstructures so as to provide a sufficiently rich set of plastic events, in view of quantifying statistically the errors made by the models. It contributes as well to bridging the gap between elementary mechanisms at the dislocation scale and the overall behavior of the polycrystalline microstructure.

\section{Experiment and modeling}

\subsection{Material and experimental setup}

As reported in our previous work [23] detailing the experimental procedure:

Bulk tensile sample (Fig. 1a) was cut from a $1.78 \mathrm{~mm}$ thickness rolled sheet of $\beta$-Ti21S alloy, produced by Titanium Metals Corporation / Toronto, Canada. The chemical composition of the material is Ti15.9Mo-2.7Nb-2.9Al-0.2Fe-0.2Si (wt \%). As a heat treatment, a soaking at $843{ }^{\circ} \mathrm{C}$ for $14 \mathrm{~min}$ was first applied before air cooling. The obtained microstructure is reported to be fully $\beta$ phase [39].

- Tensile test was performed at room temperature with a DEBEN (Suffolk, UK) machine of a maximum load cell of $1 \mathrm{kN}$. The sample was deformed under an imposed strain rate of $3.3 \times 10^{-4} \mathrm{~s}^{-1}$. Note that the unknown machine stiffness was not removed from the stressstrain curve. Therefore, the slope of the linear part (Fig. 1d) does not correspond to the Young modulus of the material. 


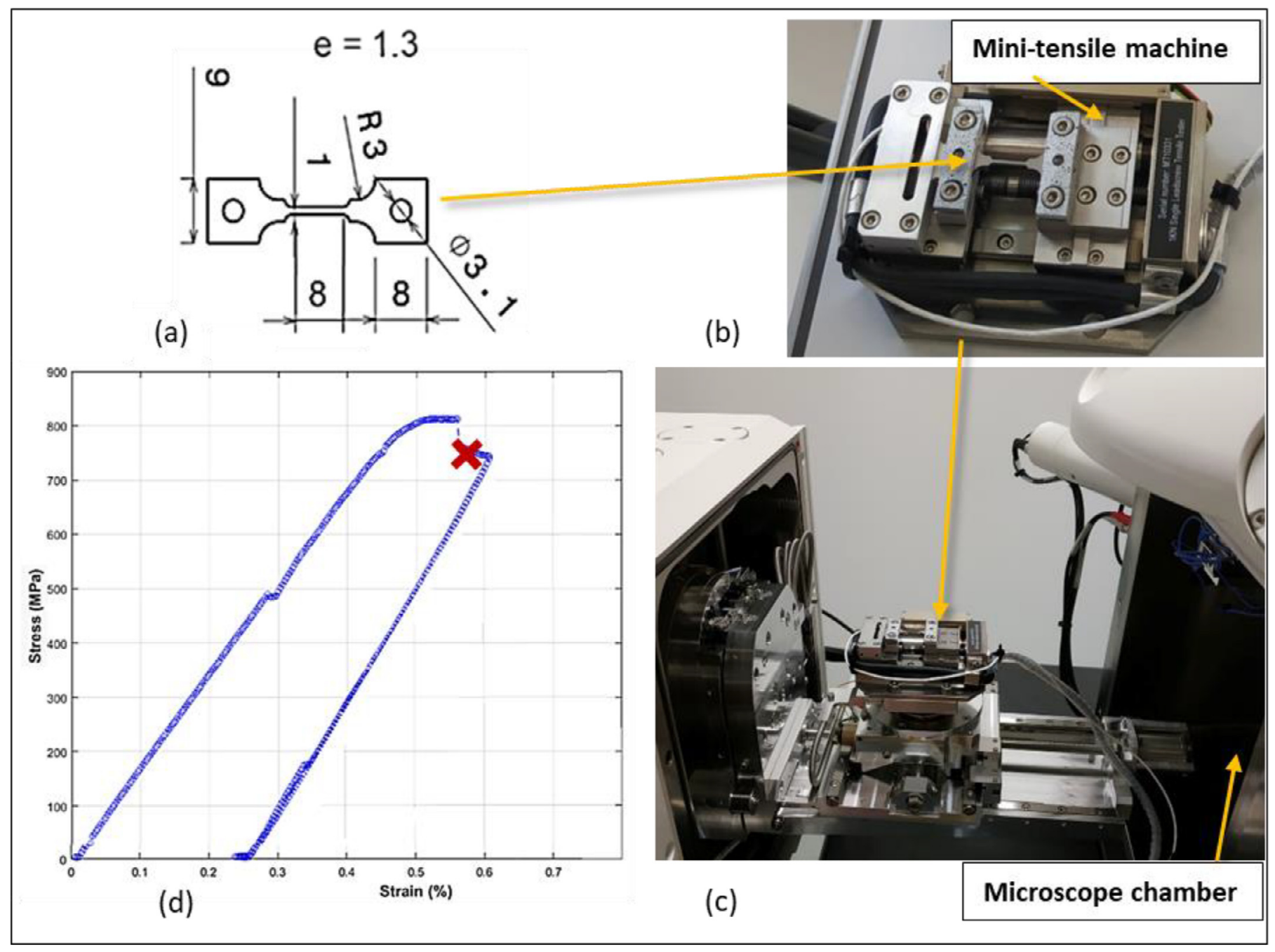

Fig. 1. Experimental setups (a) tensile specimen form and dimensions (b) specimen mounted in the mini-tensile machine (c) mounting inside the microscope (d) applied tensile curve; reported A-ECCI observations were performed at the red cross point of the curve (after 15 min relaxation).

- The stress field was confirmed to be uniaxial and homogeneous along the operational length of the sample.

- The sample was mechanically polished with $1 \mu \mathrm{m}$ diamond paste, followed by chemo-mechanical polishing with colloidal silica suspension. Finally, $2 \mathrm{~h}$ ion-polishing in a PECS II (GATAN, USA) machine was applied with a $3 \mathrm{keV}$ beam.

- Then, detailed in situ characterizations of the microstructure during deformation, were performed by A-ECCI for enabling numerous observations of defects on several and large areas of interest (Dislocations and Slip-lines), in a Zeiss Auriga Scanning Electron Microscope (SEM, Oberkochen, Germany) operating at $10 \mathrm{kV}$.

Since the BSE yield strongly depends on the orientation of the crystal relative to the incident electron beam (i.e., optical axis of the SEM), the orientation was accurately determined through High-Resolution Selected Area Channeling Patterns HR-SACP. The best defect contrast is obtained when the incident beam is oriented near a pseudo-Kikuchi band edge where the BSE yield is minimal. This corresponds to a channeling condition associated with the darkest contrast of the grain [40]. All observations were performed at constant strain.

- Prior to this step, i.e., before deformation, EBSD mapping provided grain orientations to an accuracy of about $2^{\circ}$ in a Zeiss Supra 40 SEM (Oberkochen, Germany) operating at $20 \mathrm{kV}$.

\subsection{Automated Hough transform based detection of slip line crystallography}

In order to establish an automated procedure for slip line crystallography, a trace analysis has been combined with an image analysis algorithm for slip line detection. Several experimental approaches have been widely applied for decades to study the deformation of polycrystals with a statistical significance. Yet, comparing our procedure to the existing methods was not the current work's purpose.

In the present study, the automation process is desirable not only for extracting useful statistics associated with the slip process but eventually for employing micrograph processing procedures for appropriate model selection. The ECC images were converted into a binary format with appropriate thresholding using Image-J [41], to highlight the slip lines on a given region of interest. The thresholding procedure was conducted manually to highlight the slip lines in a given region of interest and to eliminate all other sources of contrast (noise). The thresholding procedure was carried out such that maximum number of lines were contrasted in the image with minimum noise. The binary image was used in a MATLAB [42] program for automated slip line detection. The edges corresponding to each line and their corresponding pixels $(\mathrm{x}, \mathrm{y})$ are detected using a 'Canny edge detection operator' available in MATLAB. When viewed in Hough parameter space, points which are collinear in the Cartesian image space become readily apparent as they yield curves 


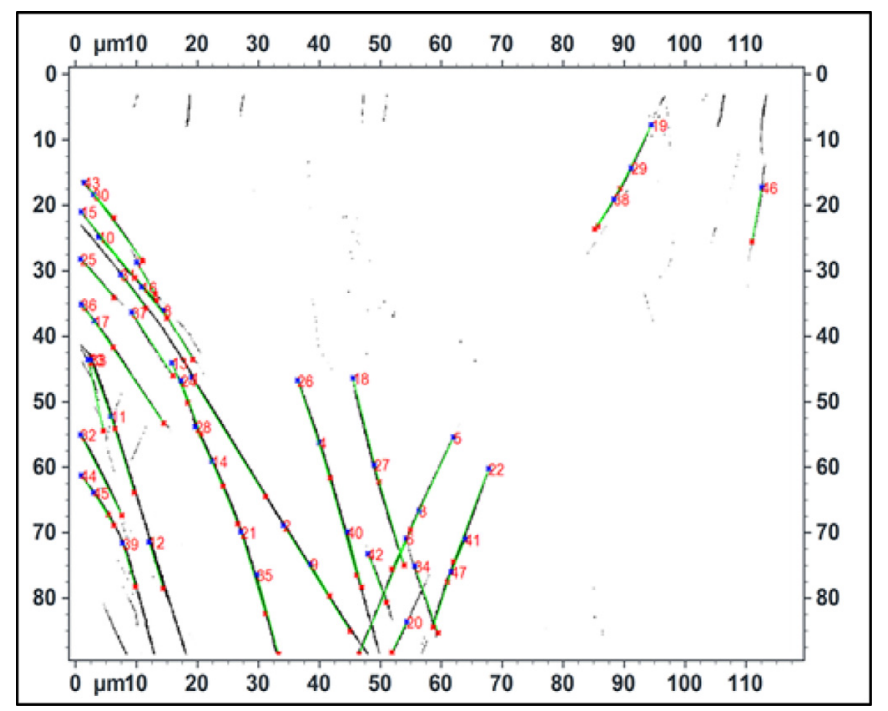

Fig. 2. Binary image used for the slip line detection algorithm.

with a common intersection point. The standard Hough transform function available in MATLAB is used on the binary image to highlight the detected slip lines. Each detected line is highlighted in green with red and blue end points and tagged by its pixel co-ordinates and a line number (Fig. 2). The angle ' $\alpha$ ' for each line (computed with respect to the loading direction) along with its pixel co-ordinates and label number are stored as an output, based on analysis of the Hough transform.

With the EBSD map of the investigated zone all the theoretical slip plane traces corresponding to each plane family ( $\{110\},\{112\},\{123\})$

1

2

3

$\left(\begin{array}{l}\mathrm{R}_{1} \\ \mathrm{R}_{2} \\ \mathrm{R}_{3}\end{array}\right)=\left(\begin{array}{c}0 \\ - \\ -\end{array}\right)$

$\left(\begin{array}{l}\mathrm{R}_{1} \\ \mathrm{R}_{2} \\ \mathrm{R}_{3}\end{array}\right)=\left(\begin{array}{c}- \\ 0 \\ -\end{array}\right)$

$\left(\begin{array}{l}\mathrm{R}_{1} \\ \mathrm{R}_{2} \\ \mathrm{R}_{3}\end{array}\right)=\left(\begin{array}{l}- \\ -\end{array}\right)$

Slip plane

Slip plane

Slip plane were determined using the ATEX software [43]. Their angles $(\alpha)$ with respect to the loading direction were computed at each pixel location on the EBSD microstructure and stored separately as an output file. For all the lines that were detected, using the Hough transform algorithm, the angular difference $\left(\left|\alpha_{1}-\alpha_{2}\right|\right)$ between the experimental slip line angle and the theoretical angle of a given slip plane family is used to determine the most consistent slip plane family. A correlation coefficient was set up taking into account the ambiguity in determination.

The correlation coefficient for each detected line, based on the angular difference can be defined as follows:

$\overrightarrow{\mathrm{R}}=\left(\begin{array}{l}\mathrm{r}_{1} \\ \mathrm{r}_{2} \\ \mathrm{r}_{3}\end{array}\right)=\left(\begin{array}{l}\cos \left[\min \left|\alpha_{1}-\alpha_{2}\right|_{\{110\}}\right] \\ \cos \left[\min \left|\alpha_{1}-\alpha_{2}\right|_{\{112\}}\right] \\ \cos \left[\min \left|\alpha_{1}-\alpha_{2}\right|_{\{123\}}\right]\end{array}\right)$

With $|\alpha 1-\alpha 2| \in\left[0, \frac{\pi}{2}\right]$.

For some of the detected lines, there can be an ambiguity in determining the most consistent slip plane family $\{110\},\{112\}$ or $\{123\}$. To account for such ambiguities, a modified correlation vector is defined as follows:

$\left(\begin{array}{l}\mathrm{R}_{1} \\ \mathrm{R}_{2} \\ \mathrm{R}_{3}\end{array}\right)=\left|\left(\begin{array}{l}\mathrm{r}_{1} \\ \mathrm{r}_{2} \\ \mathrm{r}_{3}\end{array}\right)-\left(\begin{array}{l}\max \left(\mathrm{r}_{1}, \mathrm{r}_{2}, \mathrm{r}_{3}\right) \\ \max \left(\mathrm{r}_{1}, \mathrm{r}_{2}, \mathrm{r}_{3}\right) \\ \max \left(\mathrm{r}_{1}, \mathrm{r}_{2}, \mathrm{r}_{3}\right)\end{array}\right)\right|$

This will return the best matching $R_{i}(i=1,2,3)$ as zero and others to a non-zero value. A threshold can now be defined on the $R_{i}$ 's, with $\mathrm{R}_{\mathrm{i}}<0.001$, corresponding to an angular difference of less than $3^{\circ}$. The value corresponding to $R_{i}<$ 'threshold' is set to zero, thus classifying that particular line, ' $\mathrm{i}$ ' as belonging to either two of the three possible slip systems.

According to the given convention, a detected slip line can belong to one of the six possible cases:
4

$\left(\begin{array}{l}\mathrm{R}_{1} \\ \mathrm{R}_{2} \\ \mathrm{R}_{3}\end{array}\right)=\left(\begin{array}{c}0 \\ 0 \\ -\end{array}\right)$

$\left(\begin{array}{l}\mathrm{R}_{1} \\ \mathrm{R}_{2} \\ \mathrm{R}_{3}\end{array}\right)=\left(\begin{array}{c}0 \\ - \\ 0\end{array}\right)$

$\left(\begin{array}{l}\mathrm{R}_{1} \\ \mathrm{R}_{2} \\ \mathrm{R}_{3}\end{array}\right)=\left(\begin{array}{l}- \\ 0 \\ 0\end{array}\right)$
Slip plane

\section{Slip plane}
$\{110\}$ or $\{112\}$
$\{110\}$ or $\{123\}$
$\{112\}$ or $\{123\}$

Based on this, an algorithm is formulated for counting the number of slip lines belonging to each slip system family and each detected slip line is assigned one of the six possible vectors:

\begin{tabular}{|c|c|c|c|c|c|}
\hline 1 & 2 & 3 & 4 & 5 & 6 \\
\hline$\left(\begin{array}{l}n\left(R_{1}\right) \\
n\left(R_{2}\right) \\
n\left(R_{3}\right)\end{array}\right)=\left(\begin{array}{l}1 \\
0 \\
0\end{array}\right)$ & $\left(\begin{array}{l}n\left(R_{1}\right) \\
n\left(R_{2}\right) \\
n\left(R_{3}\right)\end{array}\right)=\left(\begin{array}{l}0 \\
1 \\
0\end{array}\right)$ & $\left(\begin{array}{l}n\left(R_{1}\right) \\
n\left(R_{2}\right) \\
n\left(R_{3}\right)\end{array}\right)=\left(\begin{array}{l}0 \\
0 \\
1\end{array}\right)$ & $\left(\begin{array}{l}n\left(R_{1}\right) \\
n\left(R_{2}\right) \\
n\left(R_{3}\right)\end{array}\right)=\left(\begin{array}{c}1 / 2 \\
1 / 2 \\
0\end{array}\right)$ & $\left(\begin{array}{l}n\left(R_{1}\right) \\
n\left(R_{2}\right) \\
n\left(R_{3}\right)\end{array}\right)=\left(\begin{array}{c}1 / 2 \\
0 \\
1 / 2\end{array}\right)$ & $\left(\begin{array}{l}n\left(R_{1}\right) \\
n\left(R_{2}\right) \\
n\left(R_{3}\right)\end{array}\right)=\left(\begin{array}{c}0 \\
1 / 2 \\
1 / 2\end{array}\right)$ \\
\hline Slip plane & Slip plane & Slip plane & Slip plane & Slip plane & Slip plane \\
\hline$\{110\}$ & $\{112\}$ & $\{123\}$ & $\{110\}$ or $\{112\}$ & $\{110\}$ or $\{123\}$ & $\{112\}$ or $\{123\}$ \\
\hline
\end{tabular}

The total number of slip traces corresponding to each slip plane family and the probability of observing a slip line corresponding to a given 
slip plane family $\mathrm{P}\left[\mathrm{N}\left(\mathrm{R}_{\mathrm{i}}\right)\right]$ is defined as:

$\left(\begin{array}{l}\mathrm{N}\left(\mathrm{R}_{1}\right) \\ \mathrm{N}\left(\mathrm{R}_{2}\right) \\ \mathrm{N}\left(\mathrm{R}_{3}\right)\end{array}\right)=\sum_{\mathrm{k}=1}^{\mathrm{k}=\mathrm{M}}\left(\begin{array}{l}\mathrm{n}\left(\mathrm{R}_{1}\right) \\ \mathrm{n}\left(\mathrm{R}_{2}\right) \\ \mathrm{n}\left(\mathrm{R}_{3}\right)\end{array}\right)_{\mathrm{k}}$

for $\mathrm{M}$ slip lines detected in a given region of interest.

$\mathrm{P}\left[\mathrm{N}\left(\mathrm{R}_{\mathrm{i}}\right)\right]_{\text {for } \mathrm{i}=1,2,3}=\frac{\mathrm{N}\left(\mathrm{R}_{i}\right)}{\mathrm{N}\left(\mathrm{R}_{1}\right)+\mathrm{N}\left(\mathrm{R}_{2}\right)+\mathrm{N}\left(\mathrm{R}_{3}\right)}$

Probabilities are shown in pie charts (Fig. 5). The uncertainties are estimated using Cochran statistics [44]. This is generally used for calculating the minimum sampling size $\left(n_{\alpha}\right)$ for a representative statistic:

$\mathrm{n}_{\alpha}=\frac{\mathrm{Z}_{\mathrm{C}}^{2} \mathrm{p}_{\alpha} \mathrm{q}_{\alpha}}{\mathrm{m}_{\alpha}^{2}}$

Where $Z_{C}$ is the selected critical value of desired confidence level, $C$, obtained from the $Z_{C}$-score tables; $p_{\alpha}$ is the proportion of the glide plane family $\alpha$ according to the random distribution $\left(p_{\{123\}}=0.5\right.$; $\left.p_{\{110\}}=p_{\{112\}}=0.25\right) ; q_{\alpha}=1-p_{\alpha}$ and $m_{\alpha}$ is the desired margin of error for the glide plane family $\alpha$.

In our study, $N=123$ plastic events were tracked. Therefore, the sampling will be relevant statistically if $N \geq n_{\alpha}$.

The table below shows the best compromise for the Cochran parameters considering our sampling sizes:

\begin{tabular}{lll}
\hline Parameters & Values & \\
\hline$N$ & 123 & \\
$C$ & $95 \%$ & \\
& $\{123\}$ & $\{110\} ;\{112\}$ \\
$p_{\alpha}$ & 0.5 & 0.25 \\
$m_{\alpha}$ & $9 \%$ & $8 \%$ \\
$n_{\alpha}$ & 96 & 72 \\
\hline
\end{tabular}

Therefore, this estimation of uncertainties has to be understood as follows: one is sure at $95 \%$ that the true percentage of $\{123\}$ is localized between $[48 \%-9 \% ; 48 \%+9 \%]=[39 \% ; 57 \%]$, where $48 \%$ is the probability taken from Fig. 5.

\subsection{Brief description of the polycrystal plasticity models}

As far as the simulation part is concerned, we performed calculations according to several numerical models to predict plastic activity (activated slip systems), with direct input from EBSD map of the same deformed zone.

For comparison purposes, the paper focused on the four following polycrystal plasticity models using different assumptions, which are here summarized:

1 The mean-field model using the Sachs hypothesis (uniform stress assumption) [24]. The local 3D Cauchy stress tensor is simply set equal to the macroscopic tensile stress (only one non-zero component for uniaxial tension). With this model, each point in the microstructures can be considered independently of all others and elastic/plastic interactions between grains are neglected. More importantly, only the effect of each grain crystallographic orientation through the Schmid tensor has an effect on the projection of the stress state onto each slip systems. Note that, as aforementioned, the Sachs hypothesis is commonly used in microscopy when studying dislocation activity.

2 The Visco-Plastic Self-Consistent (VPSC) model with a "tangent linearization" of the viscoplastic flow rule [45-47]. It has been used extensively in the literature. It predicts well in particular the evolution of crystallographic textures in polycrystals at large strains. Although this model would normally be compared with experimental data in the plastic domain (ignoring the elastic part of deformation), we applied it at the onset of plasticity.

3 The Elasto-Visco-Plastic Self-Consistent (EVPSC) mean field homogenization scheme solved by the "translated field" (TF) method with an "affine linearization" of the viscoplastic flow rule [28]. Here, the constitutive equations are the same as in the recent contribution of Lhadi et al. [29], where it was applied to a fully $\beta$-Ti alloy. Here, we used the EVPSC model for the present $\beta$-Ti21S alloy, considering spherical grains with crystallographic orientations extracted from EBSD data, and compared its predictions with the other two mean-field models. In particular, elastic anisotropy is included in the EVPSC model, such that internal stresses due to both elastic and plastic anisotropies are considered. Therefore, it is expected to improve predictions with respect to the two previous mean field models.

4 Full-field polycrystal plasticity FFT (CPFFT) [48]. This elastoviscoplastic model should give the best predictions because it includes elastic anisotropy and it solves locally the balance of Cauchy stress tensor at each grid point in the loaded microstructure. Although all models are applied to the microstructure digitized from the EBSD data, this model is the best at capturing the long-range elastic strains and thus stresses due to grain interactions in the microstructure. The simulations were performed using the EBSD maps as direct input. Accordingly, they corresponded to 2D simulations with no buffer zone and strictly periodic boundary conditions. Therefore, the obtained microstructure was constituted of columnar grains (i.e., elongated along the normal direction).

\subsection{Comparison procedure}

Using EBSD data of the same zone of interest (the zone is shown in Fig. 3 and contains 110 grains), polycrystalline plasticity calculations were done as follows:

- For EVPSC and CPFFT models: the strain hardening parameters were adjusted to fit the experimental tensile curve and used the same elastic parameters. Local stress tensors were extracted for each grain point and were used as MATLAB input data to finally map the RSS and the associated slip systems. The mapping procedure is described below.

- For Sachs model, the local stress tensor was taken for each grain point equal to the macroscopic stress associated with uniaxial tensile test (only $\sigma_{11} \neq 0$ ).

- For VPSC model, all the steps from simulation to mapping were integrated in an in-house code, ATEX [43].

The local stress tensors were obtained in the sample coordinate system. With ATEX and MATLAB codes and as illustrated in Fig. 3, they were rotated in the crystal coordinate system then multiplied by the Schmid tensor to get the RSS associated with slip systems. Then, using MATLAB and ATEX codes, $1^{\text {st }}$ maximum RSS, $2^{\text {nd }}$ maximum RSS... until $\mathrm{n}^{\text {th }}$ maximum RSS maps could be generated, as well as the corresponding slip system maps. We chose to consider the n maximum RSS values that are less than $10 \%$ difference from the $1^{\text {st }}$ maximum RSS value. This percentage corresponds to the threshold value above which all models give $100 \%$ correct predictions for all active slip planes. In other terms, if we consider the $\mathrm{n}^{\text {th }}$ maximum RSS different from the $1^{\text {st }}$ maximum RSS by more than $10 \%$ (of the latter value), the four models can predict all the experimental slip planes. But below $10 \%$, the models start to be unable to predict some of the slip events.

To better illustrate this criterion, an "ambiguity map" was generated with each maximum RSS map (starting from the $2^{\text {nd }}$ ). The pixels associated to an RSS value higher than $10 \%$ of the $1^{\text {st }}$ maximum RSS value are colored in black, the rest of pixels are colored in white.

For the studied material $\beta$-Ti21S there are 48 possible slip systems belonging to three slip plane families: $\{110\}$ (12 slip systems), $\{112\}$ (12 slip systems) and \{123\} (24 slip systems) [49]. This motivated showing, for each maximum RSS map, three slip system maps corresponding to each of the three plane families. 

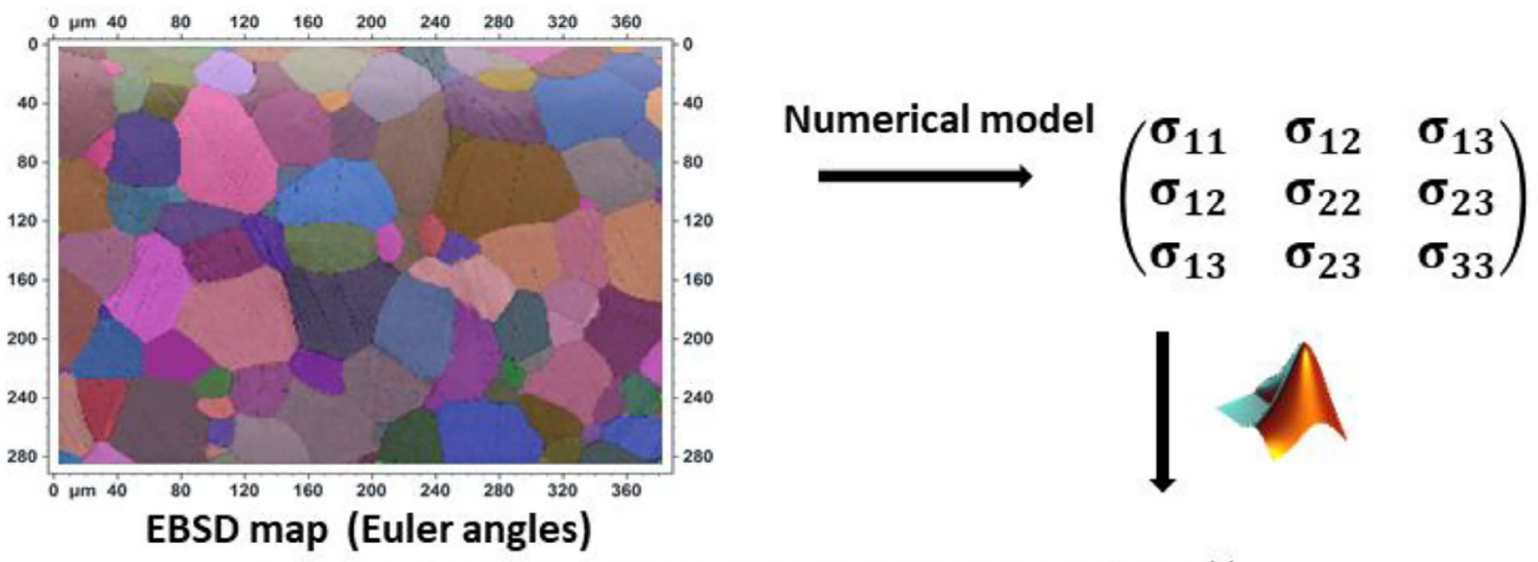

The resolved shear stress (RSS) of slip system (s): $\tau$ (s)

$$
\tau^{(s)}=\sum_{i} \sum_{j} \mathbf{m}_{i j}^{(s)} \sigma_{i j}^{c}
$$
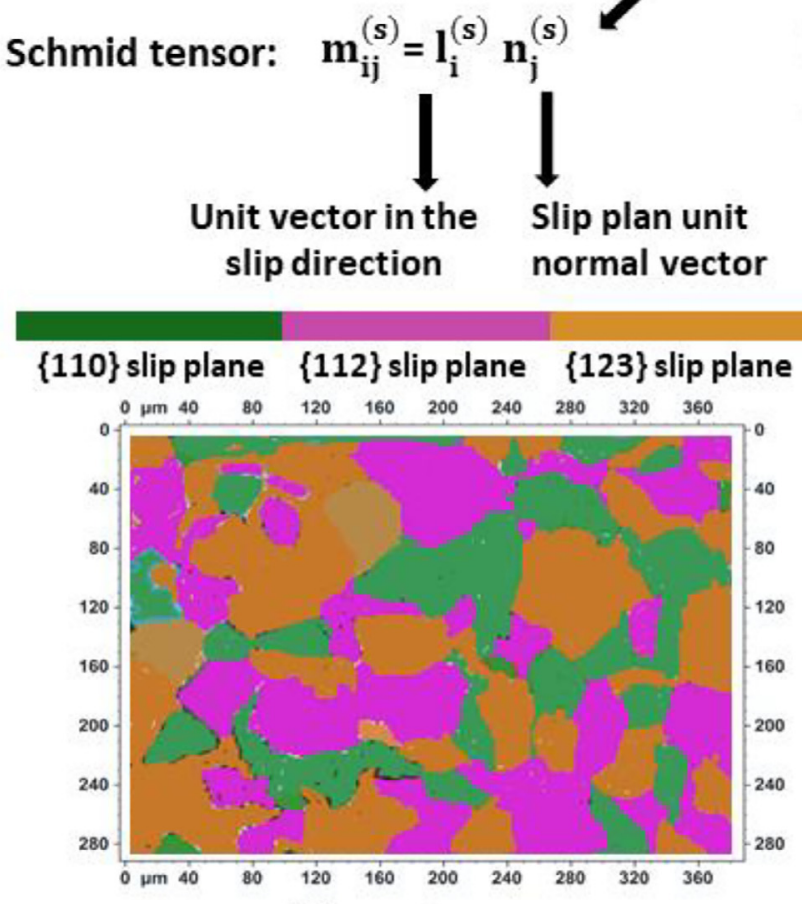

Slip system map

\section{Local stress at the crystal reference}

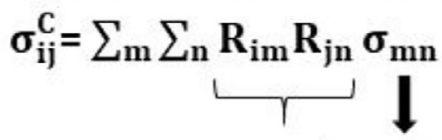

\section{Bunge Rotation Local stress at the matrix sample reference}

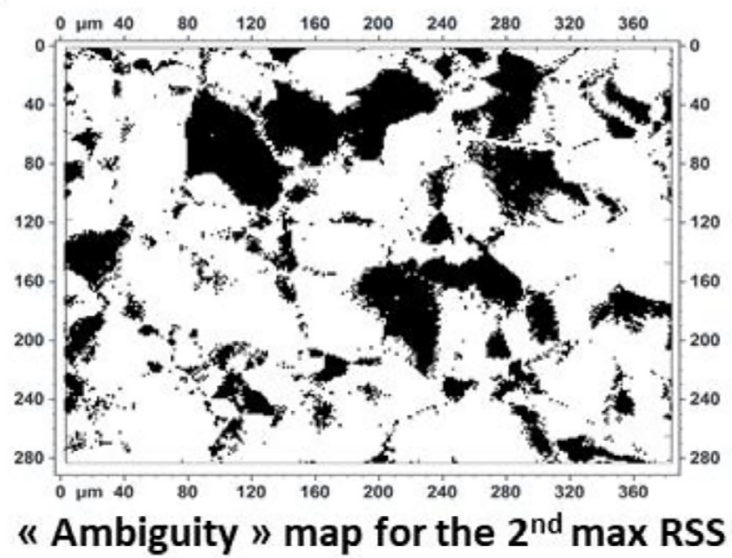

Fig. 3. Numerical calculation path from EBSD map to slip system map. (Technically speaking, "R" is a matrix constructed from the Eulers that performs transformation of axes from sample to crystal, not rotation).

\section{Results}

\subsection{Slip trace analysis}

Using ECC micrographs obtained during the in situ tensile test, a slip trace analysis was performed. The analysis was done at the onset of plasticity (curve in Fig. 4a) to determine the elementary mechanisms involved in the first steps of the deformation. After yielding and a few percent strain, the resulting microstructure is slightly deformed. Such a microstructure is investigated using A-ECCI to maximize the contrast from slip lines and dislocations [23].

The studied area from the tensile sample was chosen initially to cover slightly more than 100 grains, to allow a statistical study and enough data for micromechanical simulations using a statistically relevant region based on this sufficiently large number of grains. For detailed experimental observations and characterizations, ten particular regions of interest (each with a few grains) were selected. The examples reported in this communication correspond to a representative microstructure of 

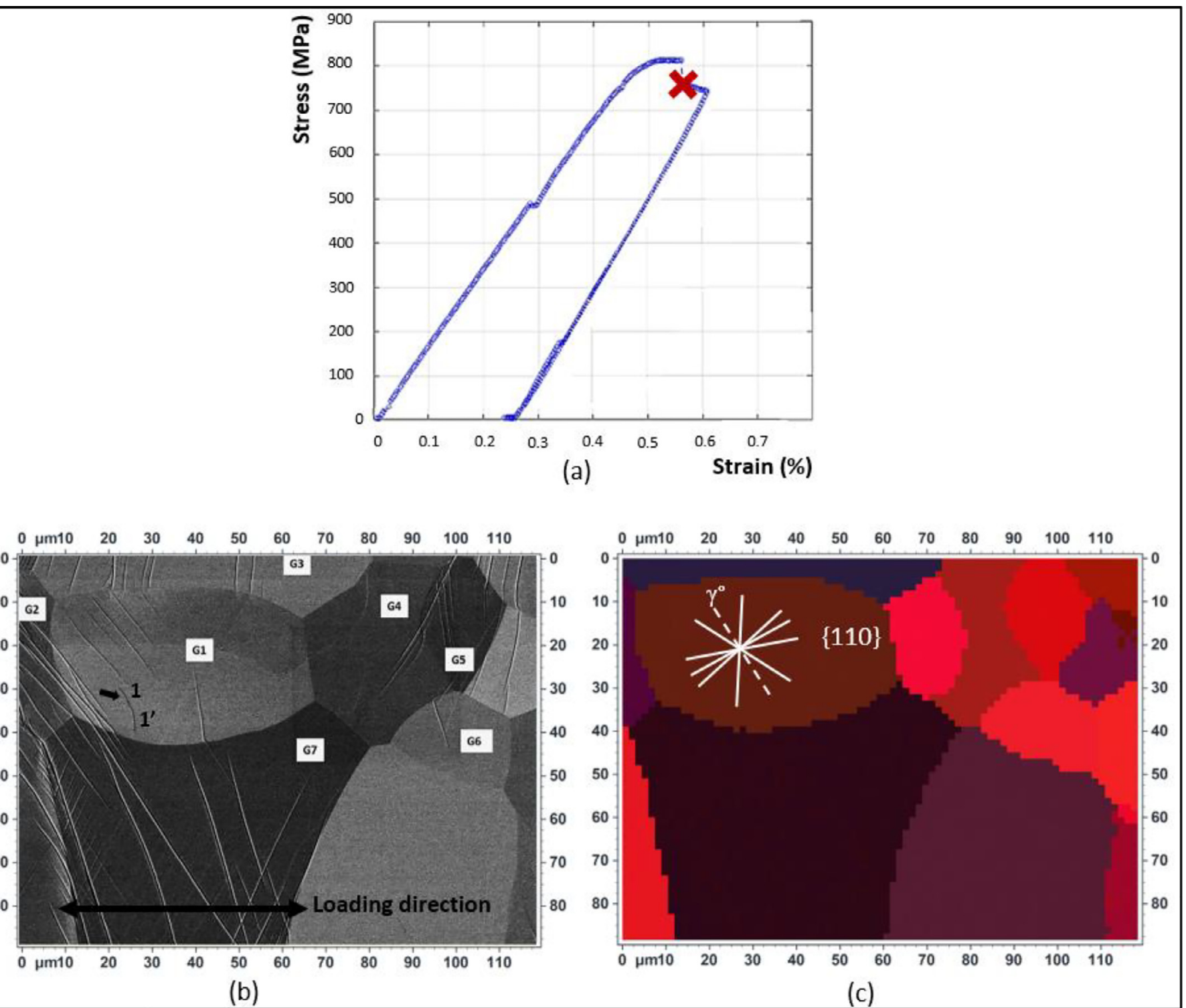

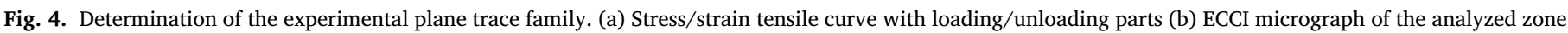
showing the slip trace segments [1] and [1'] from grain G1. (c) Theoretical slip plane traces of $\{110\}$ associated to G1 crystallographic orientation.

the whole sample, allowing to explain the procedure and to discuss the main results.

Fig. 4b shows the example region, loaded to a maximum stress of $810 \mathrm{MPa}$, that contains seven grains of interest and 55 plastic events (dislocations and slip lines). The slip trace analysis associates a slip plane type, $\{110\},\{112\}$ or $\{123\}$, to each event observed. This is done using the local orientation from EBSD data, based on which one can superimpose all possible slip systems on the slip plane trace. More precisely, the angles between the slip/dislocation lines and the loading direction (i.e., with the horizontal) were first measured. Then, using the ATEX software [43] and local orientation from the EBSD map of the investigated region as inputs, all the theoretical slip plane traces corresponding to each plane family were determined, as well as their angles to the loading direction. The smallest angle between a theoretical trace and the experimental one selects the most consistent slip plane family.

According to much previous literature, BCC plastic activity is governed by a pencil glide mechanism that does not involve a well-defined glide plane [50]. That is because in BCC crystals, $\{110\},\{112\}$ and $\{123\}$ planes have comparable atomic packing densities and all contain $<111>$ directions. Therefore, $<111>$-dislocations can slip into each of these glide planes [51]. It turns out that dislocations can easily change their slip planes, i.e., cross-slip during deformation. The dislocations move from one slip plane to another forming a stair-like shape, which results in a curved appearance in the slip trace [23,52].

Fig. $4 \mathrm{~b}$ shows the example for one curved line in grain G1 [1;1'] exhibiting pencil glide. The theoretical $\{110\}$ (Fig. $4 c$ ), $\{112\}$ and $\{123\}$ plane traces are given by the ATEX software.

For instance, line [1] forms an angle closer to $\gamma^{\circ}$ with respect to the horizontal. Calculating the differences with the real values of angles gives the closest expected slip plane family (smallest difference). Taking into consideration the angular resolution given by EBSD $\left(2^{\circ}\right)$ and the uncertainty of measuring the experimental angle $\left(\sim 1^{\circ}\right)$, the slip plane family is determined at $3^{\circ}$ of precision.

To automate the above slip trace analysis, the algorithm described in Section 2.2 was run on a total of 123 detected slip events and the probability distribution for the same is shown in Fig. 5. The results show that the majority of observed slip traces belong to the $\{123\}$ family. Besides, one can note that the statistical distribution between the three slip plane families $\{100\}, 112\}$ and $\{123\}$ (almost $25 \% / 25 \% / 50 \%$ ), roughly reflects their respective multiplicity $(12 / 12 / 24)$. 


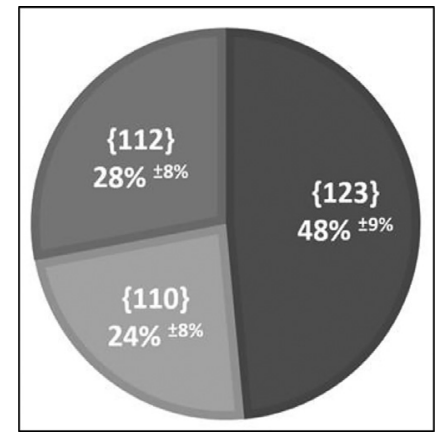

Fig. 5. Pie chart showing the probability distribution of possible slip plane families using the slip line detection algorithm for the total of 123 slip events.

\subsection{Statistical comparison of polycrystal plasticity models with experimental data}

In order to evaluate the capability of each polycrystal plasticity model in predicting the experimentally observed slip lines, we followed an approach based on Resolved Shear Stress (RSS) analysis. We tested the predictions of the four models introduced in Section 2.3, with expected increasing fraction of successful predictions: Sachs [24], VPSC [45], EVPSC [28], and CPFFT [47].

Our comparative method allowed first, to statistically quantify the difference in the deduced parameters between the four models. Fig. $6 \mathrm{~b}$ shows an example of quantification of the maximum RSS (MRSS) obtained with the Sachs and the CPFFT models (the model hypotheses are detailed in Section 2.3). The local differences are not negligible; they are the highest especially near grain boundaries at which the physical properties are discontinuous, and they range up to $100 \mathrm{MPa}$.

Then, a comparison of the RSS values obtained by each model at the beginning of the plastic domain was performed in order to identify (i) which potentially active slip system corresponds to the maximum RSS and (ii) if the latter is higher than the CRSS i.e., if the Schmid law is locally respected. To do so, after determining the experimentally observed slip plane families for the studied 55 plastic lines, a mapping of the RSS associated with each slip system (48 slip systems for BCC crystals) was performed. Then, we compared the activated slip systems predicted by each model (according to the mapping procedure described in Section 2.4) with the experimental results. Fig. $6 \mathrm{c}$ presents an example of comparison between experiments and CPFFT predictions. The first map (showing the center grain in green) is obtained by considering the $1^{\text {st }}$ MRSS and the second map (showing the middle grain in orange) is obtained by considering the $2^{\text {nd }}$ MRSS. Plane traces for lines 1 and 1 ' were experimentally determined to belong to $\{110\}$ and $\{123\}$ respectively. CPFFT calculations gave a $1^{\text {st }}$ MRSS value corresponding to $\{110\}$ slip plane and a $2^{\text {nd }}$ MRSS value corresponding to $\{123\}$ slip plane. The $2^{\text {nd }}$ MRSS value is retained since it was not very different from the $1^{\text {st }}$ MRSS (less than $10 \%$ ). Thus, we can reasonably say that the model predicts the appropriate slip plane family activation for both slip events.

The results obtained by applying the comparison procedure for the four models, show that the CPFFT and affine EVPSC give -as expectedbetter prediction than the Sachs (uniform stress) and the VPSC meanfield models. Yet, the difference in the well-predicted event percentage was quite small (86 \% up to $90 \%$ i.e., error percentage decreased from $14 \%$ to $10 \%$ ); the statistics are summarized in Table 1 .

These four different models give different incompatibility stresses with different mechanical interactions between grains. Among the four models, the Sachs model does not include internal stresses: only the macroscopic stress is considered and the incompatibility stresses are zeros. In the VPSC model, only plastic incompatibilities are included: it does not describe elastic incompatibilities owing to material's anisotropic elasticity. Both the CPFFT and the EVPSC models include
Table 1

Statistics of predicted slip events in agreement with experimental determination.

\begin{tabular}{ll}
\hline & $\begin{array}{l}\text { Error percentage of } \\
\text { predictions Vs } \\
\text { experimental results }\end{array}$ \\
\hline Model & $14 \%$ \\
VPChS (uniform stress) & $14 \%$ \\
EVPSC (mean field) & $10 \%$ \\
CPFFT (full field) & $12 \%$ \\
\hline
\end{tabular}

elastic and plastic incompatibilities in their formulations. Therefore, they give the best predictions among the different models about plastic activities in the elastic-plastic transition.

Comparison between MRSS and CRSS was made for the two models: EVPSC and CPFFT. For the first model, they were almost identical for all three slip plane families. For the second model, the CRSS was higher for $\{112\}$ and $\{123\}$ families (close values for both), than for the $\{110\}$. For both models, CRSS did not vary much compared to the initial value which is as expected because essentially no plastic slip was accumulated. Moreover, all MRSS corresponding to the activated slip planes were higher than the associated CRSS. From a statistical point of view, and for small strains, the Schmid law seems to be satisfied for this BCC material.

The same statistics as the ones reported in Table 1 were obtained for the analysis of shear rates and also when considering MRSS/CRSS values. Consequently, all interpretations and discussions made on RSS analysis can be drawn for shear rates and MRSS/CRSS fraction analysis too.

\section{Discussion}

Due to the SEM techniques used (2D EBSD and ECCI), all the reported results correspond to surfaces.

For relevant comparison, all the experimental observations as well as the numerical calculations were performed at the mesoscale.

For the studied material, $\beta$-Ti21S alloy, $\{123\}$ and $\{112\}$ slip-planes form $76 \%$ of the experimentally observed traces, against only $24 \%$ for $\{110\}$. This result is unexpected. The literature reports mainly $\{110\}$ slip-planes for experiments with single crystals. Some of our $\{123\}$ observations could be related to the surrounding microstructure in a polycrystal. In this context, Castany et al. [53] reported dislocation glide on \{123\} during an in situ TEM study of polycrystalline Ti alloy. In other cases, the observation of $\{112\}$ and $\{123\}$ can as well be explained by the fact that what we observed were "apparent" slip-planes, since the observations were made at meso-scale. These "apparent" $\{112\}$ and $\{123\}$ slip-planes may, at a finer scale, be sequences of short $\{110\}$ segments. In fact, an atomic-scale investigation by Douat et al. [54, 23], showed that microscopic $\{112\}$ slip traces in Nb BCC structure are composed of periodic sequences of $\{110\}$ traces detected by STM. In situ TEM investigations have also highlighted that dislocations were in some cases cross-slipping between different $\{110\}$ close in average to a (123) plane [55]. Even in the latter case, the experimental and theoretical results can still be compared since they were obtained both at the mesoscale.

For small strains, the Schmid law appears to be statistically satisfied. However, the Schmid law was not applicable locally for several cases, and especially not near grain boundaries, but in average it was applicable in a high fraction of cases. Yet, the tension/compression asymmetry of the yield stress is a good indicator of the presence of non-Schmid effects; this aspect, however, was not investigated here.

Concerning the critical resolved shear stress (CRSS) values, the EVPSC and CPFFT models gave different results; for the first model, CRSS was almost identical for all three slip plane families, while for the second, the CRSS was higher for $\{112\}$ and $\{123\}$ families. In the literature, contradictory results have been reported concerning CRSS values 


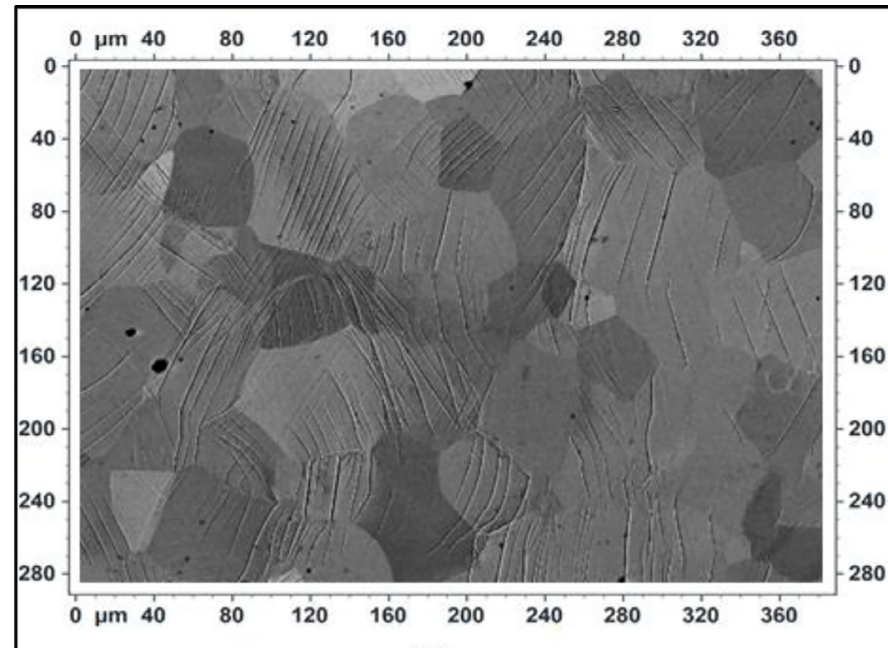

(a)

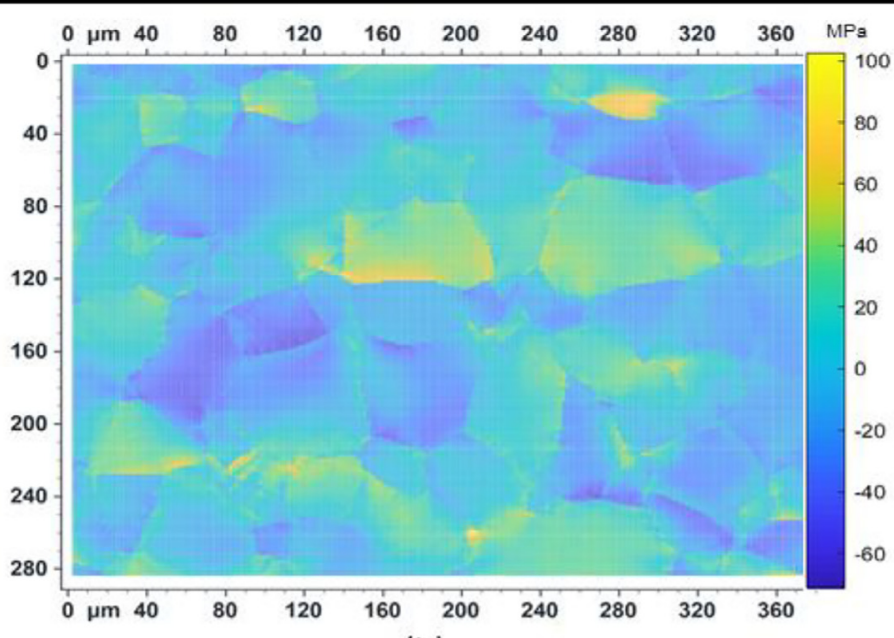

(b)
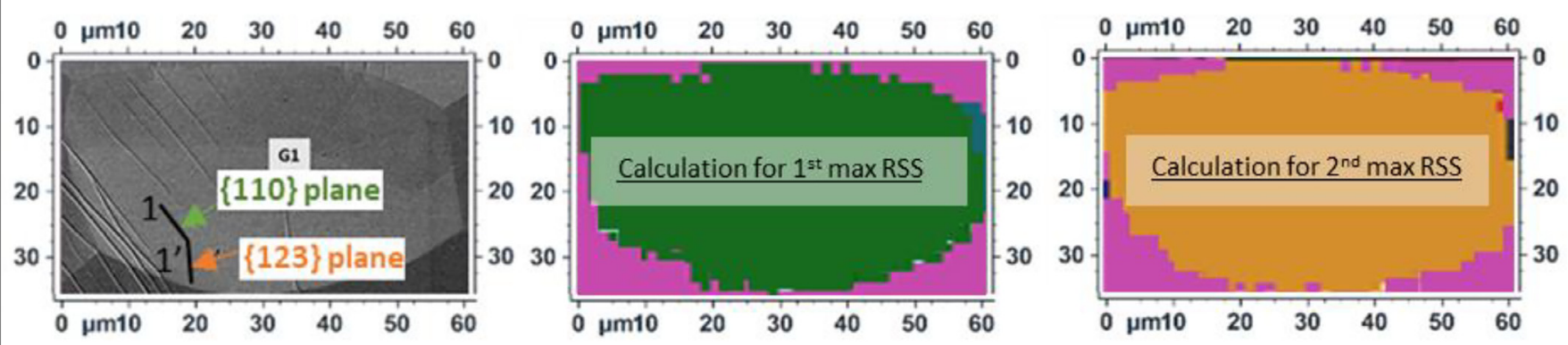

$\{110\}$ slip plane $\{112\}$ slip plane $\{123\}$ slip plane

(c)

Fig. 6. Example of comparison between experimental observations and modeling. (a) ECC image of the large area of interest (b) the map $\Delta M R S S=$ $M R S S^{F F T-E V P}-M R S S^{S a c h s}$ of the same zone (c) ECC image of grain G1 and slip plane maps according to the associated color code: in green grains where $\{110\}$ slip families were activated, in fuchsia grains where $\{112\}$ slip families were activated and in orange grains where $\{123\}$ slip families were activated.

in BCC structures. $\{112\}$ slip itself was reported to have a higher CRSS in the anti-twinning direction than in the twinning direction [56,57]. Compared with $\{110\}$ slip, $\{112\}$ slip was reported to exhibit higher CRSS in the anti-twinning direction but similar one for slip in the twinning direction [57]. Other authors stated that the CRSS of $\{112\}$ slip plane family in general is lower than that of the $\{110\}$ one [35]. Here, it raises the question as to which key parameters control the CRSS values of each slip family in BCC crystals.

Although the different models compared above are based on different assumptions and take different microstructural parameters into account (e.g. grains are considered spherical in EVPSC calculations, whereas grains are elongated in the normal direction in CPFFT calculations), they provided good predictions at the onset of plasticity. Nevertheless, for more deformed states, lower efficiency of these models is expected, which can be confirmed (or not) by applying the approach described in this paper. Moreover, to be more rigorous, a reliable model must not only consider the interactions of the crystals with their neighboring grains [58], but also must include additional hypothesis for material behavior particularly for grain boundary-adjacent areas (different from those used for the rest of microstructure i.e., inside the grains). The latter is mandatory for the study of materials with very small grain sizes, and more complex microstructures. The procedure reported here can be applied to test how the activation of slip systems depends on the values of elastic parameters $\left(\mathrm{C}_{11}, \mathrm{C}_{12}\right.$ and $\left.\mathrm{C}_{44}\right)$ measured and proposed in the literature for $\beta$-Ti21S or near- $\beta$ Ti alloys [29], and on CRSS values that can also be better fitted under higher plastic strains.

\section{Conclusion}

In this paper, we performed a multiscale and statistical analysis of plastic activity in BCC $\beta$-Ti21S, particularly at the onset of plasticity, coupling: in situ dislocation-scale characterization of plastic deformation mechanisms during macroscopic tensile test on bulk specimen, and numerical predictions of four polycrystal plasticity models based on mean field models: Sachs, VPSC, EVPSC and full-field simulations: CPFFT.

The coupling of in situ tensile testing with ECCI microstructural characterization represents a successful combination to avoid the limitations related to non-reliable statistics. It has the advantage of allowing analysis of many zones of interest and covers a maximum area of study in one single testing with a good accuracy. The relevance and reliability of our experimental results allowed a novel comparison with four different polycrystal plasticity models. This innovative procedure opens the way to validate and calibrate mesoscale models with large and statistically relevant datasets on more complex materials.

The study reported here can also be applied to in-depth statistical understanding of dislocation transfer mechanisms across grain bound- 
aries. Moreover, the described method provides input to data mining processes, and can be easily adopted by the material science community since the observation of slip lines is accessible in all SEMs equipped with Back-Scattered Electron (BSE) detectors. This approach can be coupled to DIC for a deeper investigation of slip step height, for instance.

\section{Declaration of Competing Interest}

The authors declare that they have no known competing financial interests or personal relationships that could have appeared to influence the work reported in this paper.

\section{Acknowledgments}

This work was supported by the French State through the program "Investment in the future" operated by the National Research Agency (ANR) and referenced by ANR-11-LABEX-0008-01 (LabExDAMAS) and was also supported by the Région Grand Est. The authors would like to thank Dr. Nathalie GEY, Dr. Hana KRIAA and Dr. Jean-Jacques FUNDENBERGER for fruitful discussions, and, Dr. Julien GUYON and Dr. Jean-Sébastien LECOMTE for technical support. Anthony D. Rollett is grateful for support from the LabEx DAMAS and the Department of Energy, Basic Energy Sciences, Mechanical Behavior and Radiation Effects Program led by Dr. John VETRANO, grant number DE-SC0019096.

\section{Author Contributions}

Meriem Ben Haj Slama has contributed to the conception, design of the work, acquisition, analysis, interpretation of data, and has drafted the work. Vincent Taupin, has contributed to the conception, interpretation of data, and has substantively revised the paper. Nabila Maloufi, has contributed to the conception, design of the work, and has substantively revised the paper. Kaustubh Venkatraman, has contributed to the analysis of data and the drafting of the work. Anthony D. Rollett, Ricardo A. Lebensohn, Stéphane Berbenni and Benoît Beausir have contributed to the analysis of data and the creation of new software used in the work. Antoine Guitton, has contributed to the conception, design of the work, interpretation of data, and has substantively revised the paper.

\section{Supplementary materials}

Supplementary material associated with this article can be found, in the online version, at doi:10.1016/j.mtla.2020.100996.

\section{References}

[1] S. Ismail-Beigi, T.A. Arias, Ab initio study of screw dislocations in Mo and Ta: a new picture of plasticity in bcc transition metals, Phys. Rev. Lett. 84 (2000) 1499-1502.

[2] D. Rodney, L. Ventelon, E. Clouet, L. Pizzagalli, F. Willaime, Ab initio modeling of dislocation core properties in metals and semiconductors, Acta Mater 124 (2017) 633-659.

[3] Hong Lia, Stefan Wurster, Christian Motz, Lorenz Romaner, Claudia AmbroschDraxl, Reinhard Pippan, Dislocation-core symmetry and slip planes in tungsten alloys: Ab initio calculations and microcantilever bending experiments, Acta Mater 60 (2012) 748-758.

[4] Zongde Kou, Yanqing Yang, Lixia Yang, Bin Huang, Yanxia Chen, Xian Luo, New role of screw dislocation in twin lamella during deformation: an in situ TEM study at the atomic scale, Mater. Sci. Eng. A 729 (2018) 125-129.

[5] M. Legros, Comptes Rendus Physique (2014) 224-240.

[6] F. Mompiou, M. Legros, A. Boé, M. Coulombier, J.-P. Raskin, T. Pardoen, Inter- and intragranular plasticity mechanisms in ultrafine-grained $\mathrm{Al}$ thin films: an in situ TEM study, Acta Mater 61 (1) (2013) 205-216.

[7] S. Kahla, R. LinPeng, M. Calmunger, B. Olsson, S. Johansson, In situ EBSD during tensile test of aluminum AA3003 sheet, Micron 58 (2014) 15-24.

[8] Nicolas Bertin, Ryan B.Sills, Wei Cai, Frontiers in the simulation of dislocations, Annu. Rev. Mater. Res 50 (21) (2020) 1-28.

[9] Landon T. Hansen, David T. Fullwood, Eric R. Homer, Robert H. Wagoner, Hojun Lim, Jay D. Carroll, Guowei Zhou, Hyuk Jong Bong,, An investigation of geometrically necessary dislocations and back stress in large grained tantalum via EBSD and CPFEM, Mater. Sci. Eng. A 772 (2020) 138-704.
[10] Hongjia Zhang, Antoine Jérusalem, Enrico Salvati, Chrysanthi Papadaki, Kai Soon Fong, Xu Song, Alexander M. Korsunsky, Datasets for multi-scale diffraction analysis (synchrotron XRD and EBSD) of twinning-detwinning during tensile-compressive deformation of AZ31B magnesium alloy samples, Data in Brief 26 (2019) 104-423.

[11] Huachun Pi, Jingtao Han, Chuanguo Zhang, A.Kiet Tieu, Zhengyi Jiang, Modeling uniaxial tensile deformation of polycrystalline Al using CPFEM, Materials 15 (1) (2008) 43-47.

[12] Fabio Di Gioacchino, João Quinta da Fonseca, An experimental study of the polycrystalline plasticity of austenitic stainless steel, Int. J. Plast. 74 (2015) 92-109.

[13] N. Gao, D. Perez, G.H. Lu, Z.G. Wang, Molecular dynamics study of the interaction between nanoscale interstitial dislocation loops and grain boundaries in BCC iron, J. Nuc. Mater. 498 (2018) 378-386.

[14] Ki Jung Kim, Jang Hyuk Yoon, Min Hyung Cho, Ho Jang, Molecular dynamics simulation of dislocation behavior during nanoindentation on a bicrystal with a $\Sigma=5$ (210) grain boundary, Mater. Lett. 60 (2006) 3367-3372.

[15] F. Roters, P. Eisenlohr, L. Hantcherli, D.D. Tjahjanto, T.R. Bieler, D. Raabe, Overview of constitutive laws, kinematics, homogenization and multiscale methods in crystal plasticity finite-element modeling: theory, experiments, applications, Act. Mater. 58 (2010) 1152-1211.

[16] Julian Kochmann, Stephan Wulfinghoff, Lisa Ehle, Joachim Mayer, Bob Svendsen, Stefanie Reese, Efficient and accurate two-scale FE-FFT-based prediction of the effective material behavior of elasto-viscoplastic polycrystals, Comput. Mech. 61 (2018) 751-764.

[17] H. Kriaa, A. Guitton, N. Maloufi, Fundamental and experimental aspects of diffraction for characterizing dislocations by electron channeling contrast imaging in scanning electron microscope, Sci. Rep. 7 (2017).

[18] H. Mansour, M.A. Crimp, N. Gey, N. Maloufi, Accurate electron channeling contrast analysis of a low angle sub-grain boundary, Scripta Mater 109 (2015) 76-79.

[19] A. Guitton, H. Kriaa, E. Bouzy, J. Guyon, N. Maloufi, A dislocation-scale characterization of the evolution of deformation microstructures around nanoindentation imprints in a TiAl Alloy, Materials 11 (2) (2018) 305.

[20] H. Mansour, J. Guyon, M. Crimp, N. Gey, B. Beausir, N. Maloufi, Accurate electron channeling contrast analysis of dislocations in fine grained bulk materials, Scripta Mater 84 (2014) 11-14.

[21] J. Guyon, H. Mansour, N. Gey, M. Crimp, S. Chalal, N. Maloufi, Sub-micron resolution selected area electron channeling patterns, Ultramicroscopy 149 (2015) 34-44.

[22] G Sachs, Zur Ableitung einer Fliessbedingung, Z. VDI. 72 (1928) 734-736.

[23] Meriem Ben Haj Slama, Nabila Maloufi, Julien Guyon, Slim Bahi, Laurent Weiss, Antoine Guitton, In situ macroscopic tensile testing in SEM and electron channeling contrast imaging: pencil glide evidenced in a bulk $\beta$-Ti21S polycrystal, Materials 12 (15) (2019) 2479.

[24] A Reuss, Berechnung der Fliessgrenze von Mischkristallen auf Grund der Plastizitätsbedingung für Einkristalle, J. Appl. Math. Mech. 9 (1929) 49-58.

[25] G.I. Taylor, Plastic strains in metals, J. Inst. Met. 62 (1938) 307-324.

[26] AV Hershey, The elasticity of an isotropic aggregate of anisotropic cubic crystals, J. Appl. Mech. 21 (1954) 236-240.

[27] JW Hutchinson, Bounds and self-consistent estimates for creep of polycrystalline materials, Proc. R. Soc. Lond. A 348 (1976) 101-127.

[28] C. Mareau, S. Berbenni, An affine formulation for the self-consistent modeling of elasto-viscoplastic heterogeneous materials based on the translated field method, Int. J. Plast. 64 (2015) 134-150.

[29] S. Lhadi, S. Berbenni, N. Gey, T. Richeton, L. Germain, Micromechanical modeling of the effect of elastic and plastic anisotropies on the mechanical behavior of beta-Ti alloys, Int. J. Plast. 109 (2018) 88-107.

[30] H Moulinec, P Suquet, A fast numerical method for computing the linear and nonlinear mechanical properties of composites, C R Acad. Sci. 318 (1994) 1417-1423.

[31] H Moulinec, P Suquet, A numerical method for computing the overall response of nonlinear composites with complex microstructures, Comput. Methods Appl. Mech. Eng. 157 (1) (1998) 69-94.

[32] J.W. Christian, Some surprising features of the plastic deformation of Body-Centered Cubic metals and alloys, Metall. Trans. A 14 (1983) 1237-1256.

[33] G. Taylor, Thermally-activated deformation of BCC metals and alloys, Prog. Mater. Sci. 36 (1992) 29-61.

[34] M.S. Duesbery, V. Vitek, Plastic anisotropy in BCC transition metals, Acta Mater 46 (1998) 1481-1492.

[35] B. Orlans-Joliet, J.H. Driver, F. Montheillet, Plane strain compression of silicon-iron single crystals, Acta Metall. Mater. 38 (1990) 581-594.

[36] Lucile Dezerald, David Rodney, Emmanuel Clouet, Lisa Ventelon, François Willaime, Plastic anisotropy and dislocation trajectory in BCC metals, Nat. Comm. 7 (2016) 11695.

[37] Y. Aono, E. Kuramoto, K. Kitajima, Orientation dependence of slip in niobium single crystals at 4.2 and 77 K, Scripta Mater 18 (1984) 201-205.

[38] Z.Q. Wang, I.J. Beyerlein, An atomistically-informed dislocation dynamics model for the plastic anisotropy and tension-compression asymmetry of BCC metals, Int. J. Plas. 27 (2011) 1471-1484.

[39] L. Weiss, J. Zollinger, P. Sallamand, E. Cicala, A. Mathieu, E. Fleury, Mechanical properties and microstructural study of homogeneous and heterogeneous laser welds in $\alpha, \beta$, and $\alpha+\beta$ titanium alloys, Int. Inst. Welding 63 (2019) 53-62.

[40] P. Morin, M. Pitaval, E. Vucario, G. Fontaine, Scanning electron microscope observation of single defects in solid crystalline materials, Scanning 2 (1979) 217-224.

[41] CA Schneider, WS Rasband, KW Eliceiri, NIH image to imagej: 25 years of image analysis, Nat Methods 9 (7) (2012) 671-675.

[42] H. Benker, MATLAB. In: Ingenieurmathematik mit Computeralgebra-Systemen. Ausbildung und Studium, Vieweg+Teubner Verlag, 1998. 
[43] B. Beausir, J.-J. Fundenberger, Université de Lorraine - metz, ATEX@ - analysis tools for electron and X-ray diffraction, http://ATEX-software.eu/ (2015).

[44] William G. Cochran, Gertrude M. Cox, Experimental Designs, Wiley Inc., New York, 1957.

[45] A. Molinari, G.R. Canova, S. Ahzi, A self-consistent approach of the large deformation polycrystal viscoplasticity, Acta Metall 35 (1987) 2983-2994.

[46] R.A. Lebensohn, C.N. Tomé, A self-consistent approach for the simulation of plastic deformation and texture development of polycrystals: application to Zirconium alloys, Acta Metallurgica et Materialia 41 (1993) 2611-2624.

[47] A. Molinari, L.S. Toth, Tuning a self-consistent viscoplastic model by finite element results-I. Modeling, Acta Metall. Mater. 42 (1994) 2453-2458.

[48] RA Lebensohn, AK Kanjarla, P Eisenlohr, An elastoviscoplastic formulation based on fast Fourier transforms for the prediction of micromechanical fields in polycrystalline materials, Int. J. Plast. 32-33 (2012) 59-69.

[49] Alexis C. Lewis, Siddiq M. Qidwai, Andrew B. Geltmacher, Slip systems and initiation of plasticity in a body-centered-cubic titanium alloy, Met. Mater. Trans. A (41) (2010) 2522-2531.

[50] G.I. Taylor, F.R.S, D. C. F. Elam, The distortion of iron crystals, in: Proceedings of the Royal Society of London. Series A, Containing Papers of a Mathematical and Physical Character, 112, 1926, pp. 337-361.
[51] A. Seeger, W. Wasserbäch, Anomalous slip - a feature of high-purity body-centred cubic metals, phys. stat. sol. (a) 189 (1) (2002) 27-50.

[52] A.H.W. Ngan, M. Wen, Dislocation kink-pair energetics and pencil glide in bodycentered-cubic crystals, Phys. Rev. Lett. 87 (7) (2001) 075505.

[53] P. Castany, M. Besse, T. Gloriant, In situ TEM study of dislocation slip in a metastable $\beta$ titanium alloy, Scrip. Mater. 66 (6) (2012) 371-373.

[54] B. Douat, C. Coupeau, J. Bonneville, M. Drouet, L. Vernisse, L. Kubin, Atomic-scale insight into non-crystallographic slip traces in body-centred cubic crystals, Scripta Mater 162 (2019) 292-295.

[55] F. Mompiou, D. Tingaud, Y. Chang, B. Gault, G. Dirras, Conventional vs harmonic-structured $\beta$-Ti-25Nb-25Zr alloys: a comparative study of deformation mechanisms, Acta Materialia 161 (2018) 420-430.

[56] T. Taoka, S. Takeuchi, E. Furubayashi, Slip systems and their critical shear stress in 3 \% silicon iron, J. Phys. Soc. Jpn. 19 (1964) 701-711.

[57] P. Franciosi, L.T. Le, G. Monnet, C. Kahloun, M.H. Chavanne, Investigation of slip system activity in iron at room temperature by SEM and AFM in-situ tensile and compression tests of iron single crystals, Int. J. Plast. 65 (2015) 226-249.

[58] H. Abdolvand, J. Wright, A.J. Wilkinson, Strong grain neighbour effects in polycrystals, Nat. Comm. 9 (2018) 171 